\title{
A Class Age-Structured HIVIAIDS Model with Impulsive Drug-Treatment Strategy
}

\author{
Helong Liu and Lianbing Li \\ College of Mathematics and Information, Xinyang Normal University, Henan 464000, China \\ Correspondence should be addressed to Helong Liu, liuhelong2004@yahoo.com.cn
}

Received 28 September 2009; Accepted 28 January 2010

Academic Editor: Antonia Vecchio

Copyright (C) 2010 H. Liu and L. Li. This is an open access article distributed under the Creative Commons Attribution License, which permits unrestricted use, distribution, and reproduction in any medium, provided the original work is properly cited.

We formulate an HIV/AIDS transmission model that considers the dependence of HIV / AIDS progress on infection age (the time since infection), disease age (the time elapsed since the onset), and impulsive antiretroviral treatment. Since no effective vaccine is available for HIV/AIDS, our impulsive disease-control strategy is targeted at infected individuals (I control). Thus the model only includes infective class and AIDS class: infected population is the state at birth, and AIDS population is not the state at birth. Assuming the theoretical strategy can provide HIV testing for risk population groups every $T$ years and immediate antiretroviral treatment for HIV-positive people. The action is approximated by impulsive differential equations. We demonstrate the effect of the impulsive drug treatment and show that there exists a globally stable infection-free state when the impulsive period Tand drug-treatment proportion $p$ satisfy $R(p, T)<1$. This result shows that the prevention effects can drive HIV / AIDS epidemic towards to elimination.

\section{Introduction}

Human immunodeficiency virus (HIV) disease has become one of the major public health problems in the world. According to the figures published in Joint United Nations Program on HIV / AIDS (UNAIDS) (2007), there were about 2.7 million (2.2-3.2 million) new infections in 2007, estimated 33 million (30-36 million) people living with HIV, and 2 million (1.8-2.3 million) AIDS deaths.

As HIV is hypervariable, both within infected individuals and on a population basis, due to its rapid replication rate, high mutation rate, and capacity for recombination, there are many difficulties in developing a safe, effective, accessible vaccine to prevent HIV infection. Then, the effective ways to prevent HIV/AIDS epidemic are to use drug treatment strategies for HIV individuals. Standard antiretroviral therapy (ART) consists of the use of at least three antiretroviral (ARV) drugs to maximally suppress the HIV virus and stop the progression of HIV disease. Huge reductions have been seen in rates of death and suffering when use is made of a potent ARV regimen [1]. 
About 33 million people are now living with HIV, of whom more than 30 million live in low- and middle-income countries. WHO estimates that at least 9.7 million of these people in need of ART. As of December 2007, 3 million people had access to ART in low- and middle-income countries [2]. Due to economic and social constrains, especially in developing countries, it is impossible to make all HIV-positive individuals receive antiretroviral therapies. For HIV-positive individuals, this suggests the possibility of using mathematical models to design and evaluate drug treatment proportion in which can be prevented HIV / AIDS outbreak.

HIV virus has the long incubation and infectious periods (infection age, from 8 to 10 years). During the incubation period, the infectivity of infected people is varying depending on the time since infection. When symptom onset appears, AIDS population transmission rate depends on disease-age (i.e., the time elapsed since the onset). In fact, the host behavior and his/her contact patterns are heavily influenced by the severity of clinical symptoms. Different from common infectious diseases, the time scale of HIV/AIDS transmission is so long that demographic of the host population could affect transmission process.

A handful of age-structured or class age-structured models has been developed for HIV / AIDS [3-6]. The dynamics of these models tend to generally be completely determined by a threshold quantity called the basic reproduction number (denoted by $R_{0}$ ), which measures the expected number of secondary infections from a single individual during his or her entire infectious period, in a population of susceptibles. May and Anderson [7] found $R_{0}$ for some simple HIV transmission models. May et al. [6] used models with age structure to examine the demographic effects of AIDS in African countries. Busenberg and Castillo-Chavez [3] found an $R_{0}$ expression for an HIV model with variable infectivity, continuous chronological and HIV-class age structure, and proportionate mixing. Hyman et al. [4] generalized these results on $R_{0}$ to HIV models. Inaba [5] considered an age-durationstructured population model for HIV infection in a homosexual community, and investigated the invasion problem to establish the basic reproduction ratio $R_{0}$ for HIV / AIDS.

When a disease-control strategy is targeted at susceptible individuals (S control in [8]) in a static environment, the presence of a small fraction of asymptomatic infections may not even be a significant concern of the disease control itself. For HIV/AIDS transmission, since no vaccines is available, the target of HIV / AIDS control must be the infected individuals. So, our HIV / AIDS model only considers HIV-positive individuals and AIDS individuals.

Assuming an HIV prevention programme can provide HIV testing for risk population groups every $T$ years, and gives antiretroviral treatment to HIV-positive people. The action period is negligibly short in comparison with the incubation and infectous period of HIV/AIDS; hence, it can be approximated by impulsive differential equations. Where we neglect that identifying individuals takes time, it is more reasonable to formulate the model as a system of impulsive delay differential equations. Due to economic constraints, a fraction of the entire infective population is detected and is provided with antiretroviral treatment in a single pulse applied every $T$ years. We assume that these individuals who have received antiretroviral treatment are less likely to transmit the virus to others. The assumption is based on the facts: the people living with HIV are less likely to transmit the virus to others if they know they are infected; antiretroviral therapy lowers infectivity and also treatment may be coupled to safer sex education. On the other hand, the infectivity of infected people is varying depending on the time since infection. Hence, the infection age and disease age play an important role in the progress of HIV/AIDS. For AIDS individuals, symptom onset is clearly separated by definition, these individuals can be isolated when they show symptoms; its transmission risk is not high in general, but we could not neglect this factor. In summary, 
for HIV / AIDS model we should consider infection age, disease age, and impulsive effects. Those aspects have been often neglected in traditional HIV / AIDS models.

Fairly rich results have been achieved for the theories of impulsive differential equations for almost twenty years [9]. Some scholars have applied the theories of impulsive differential equations in some simple SIR or SIS models with pulse vaccination [10-12], which are described by impulsive ordinary differential equations. Some successful applications of the theories of impulsive differential equations are the control of poliomyelitis and measles in Central and South America and the UK vaccination campaign against measles in [11-13]. Impulsive equations have been recently introduced into some HIV transmission models in relation to impulsive drug behaviour [1, 14, 15]. Differently from those authors mentioned above, in order to reflect the dependence of HIV/AIDS progress on the infection age, disease age and impulsive antiretroviral treatment, we formulate a class age-structured population model of HIV/AIDS infection with impulsive effects, and discuss the role of impulsive proportion and impulsive period in controlling HIV/AIDS transmission. The study is instructive to find an optimal drug treatment strategy to control HIV / AIDS at least cost.

This paper is organized as follows: Section 2 introduces an HIV/AIDS model with impulsive drug treatment strategy and class age (infection age, disease age), which includes infective class and AIDS class, infected population is the state at birth, and AIDS population is not the state at birth. In Section 3, we discuss the condition in which HIV / AIDS can persist, and study global stability of infection-free state $E_{0}$, which implies that HIV/AIDS will be vastly reduced. Section 4 contains some discussions of the results.

\section{The Model}

In this section, the HIV / AIDS transmission model only includes two groups: I (HIV-infected) and $A$ (fully developed AIDS symptoms). Let $I(t, \theta)$ be the density of the HIV-infected population at infection age (i.e., the time since infection) $\theta$ and time $t$, and $A(\tau, t)$ the density of the AIDS population at time $t$ and disease age (i.e., the time since the onset of AIDS) $\tau$. Let $\mu(\xi)$ be the class age-specific mortality rate of HIV-infected population and AIDS population, respectively, $\gamma_{j}(\xi)$ the disease-induced mortality rate of HIV-infected population at infectionage $\xi$ if $j=1$ or AIDS population at disease-age $\xi$ if $j=2$.

HIV-infected individuals and AIDS individuals can transfer infection through some direct or indirect contact with susceptible individuals. However, AIDS state is not the state at birth; only HIV-infected individuals can develop AIDS individuals. Let $S_{0}$ be the initial density of the susceptible population, $\eta(\theta)$ the rate of transition from the infective class to AIDS class, which depends on infection age $\theta, \beta_{1}(\theta)$ the transmission rate of HIV-infected individuals at infection-age $\theta$, and $\beta_{2}(\tau)$ the transmission rate of AIDS individuals at diseaseage $\tau$.

Few people are aware of their HIV status; HIV testing for risk population groups is an important prevention approach for HIV/AIDS; where we use impulsive HIV testing scheme for risk population groups, and apply drug treatment scheme to infected individuals (who are not on treatment). Immediately following each impulsive drug treatment, the population evolves from its new initial state without being further affected by the drug treatment until next pulse is applied. Due to economic constraints, a fraction of all infected individuals is detected, and provided antiretroviral treatment in a single pulse. Since the drug treatment, at $t=n T, n \in N_{+}, I(t, \theta)$ is converted into $(1-p) I(t, \theta)$. 
This can be formulated as

$$
I(t, \theta)=(1-p) I(t, \theta), \quad n \in N_{+},
$$

where $p(0<p<1)$ is drug treatment proportion at which infected individuals receive antiretroviral therapies. $T$ is the impulsive period, and is allowed to be a positive real number, $n T$ is the time at which we apply the $n$th $\left(n \in N_{+}\right)$pulse, and $n T^{-}$is the time just before applying the $n$th pulse. For AIDS individuals, we use constant isolation and treatment police (continuous isolation and treatment police ), that is, as soon as an infected individual appears symptom, we isolate and treat him or her. Let $\rho$ be the isolation and treatment rate of AIDS individuals.

Then, the dynamics of the class age-structured population is governed by the following initial boundary value problem:

$$
\begin{gathered}
\frac{\partial I}{\partial t}+\frac{\partial I}{\partial \theta}=-\left(\mu(\theta)+\eta(\theta)+\gamma_{1}(\theta)\right) I(t, \theta), \quad \theta>0, t \neq n T, \\
I(n T, \theta)=(1-p) I\left(n T^{-}, \theta\right), \quad \theta>0, t=n T, \\
\frac{\partial A}{\partial t}+\frac{\partial A}{\partial \tau}=-\left(\mu(\tau)+\rho+\gamma_{2}(\tau)\right) A(t, \tau), \quad \tau>0, t \neq n T, n \in N_{+}, \\
A(n T, \tau)=A\left(n T^{-}, \tau\right), \quad \tau>0, t=n T, \\
I(t, 0)=S_{0} \int_{0}^{+\infty} \beta_{1}(\theta) I(t, \theta) d \theta+S_{0} \int_{0}^{+\infty} \beta_{2}(\tau) A(t, \tau) d \tau, \\
A(t, 0)=\int_{0}^{+\infty} \eta(\theta) I(t, \theta) d \theta,
\end{gathered}
$$

with initial conditions

$$
I(0, \theta)=I_{0}(\theta) \geq 0, \quad A(0, \tau)=A_{0}(\tau) \geq 0 .
$$

Using the perturbation method of nondensely defined operators [16, 17], similar to Appendix A of Inaba [5], we can show that system (2.2) and (2.3) is well-posed.

In order to simplify system (2.2) and (2.3), we introduce new functions $i, a$ by

$$
I(t, \theta)=l(\theta) \Gamma_{1}(\theta) \Pi_{1}(\theta) i(t, \theta), \quad A(t, \tau)=l(\tau) \Gamma_{2}(\tau) \Pi_{2}(\tau) a(t, \tau),
$$

where $l(\theta) \Gamma_{1}(\theta) \Pi_{1}(\theta)$ and $l(\tau) \Gamma_{2}(\tau) \Pi_{2}(\tau)$, are the proportion of, respectively, infecteds who remain in the asymptomatic state at infection-age $\theta$ and symptomatic cases who stay in the symptomatic class at disease-age $\tau$ defined by

$$
l(a)=e^{-\int_{0}^{a} \mu(\tau) d \tau}, \quad \Gamma_{j}(\theta)=e^{-\int_{0}^{\theta} \gamma_{j}(\tau) d \tau} \quad(j=1,2), \quad \Pi_{1}(\theta)=e^{-\int_{0}^{\theta} \eta(\tau) d \tau}, \quad \Pi_{2}(\tau)=e^{-\rho \tau} .
$$


By the transformation (2.4), we obtain that

$$
\begin{gathered}
\frac{\partial i}{\partial t}+\frac{\partial i}{\partial \theta}=0, \quad \theta>0, t \neq n T, \\
i(n T, \theta)=(1-p) i\left(n T^{-}, \theta\right), \quad \theta>0, t=n T, \\
\frac{\partial a}{\partial t}+\frac{\partial a}{\partial \tau}=0, \quad \tau>0, t \neq n T, n \in N_{+}, \\
a(n T, \tau)=a\left(n T^{-}, \tau\right), \quad \tau>0, t=n T, \\
i(t, 0)=S_{0} \int_{0}^{+\infty} \beta_{1}(\theta) l(\theta) \Gamma_{1}(\theta) \Pi_{1}(\theta) i(t, \theta) d \theta+S_{0} \int_{0}^{+\infty} \beta_{2}(\tau) l(\tau) \Gamma_{2}(\tau) \Pi_{2}(\tau) a(t, \tau) d \tau, \\
a(t, 0)=\int_{0}^{+\infty} \eta(\theta) l(\theta) \Gamma_{1}(\theta) \Pi_{1}(\theta) i(t, \theta) d \theta,
\end{gathered}
$$

with initial conditions

$$
i(0, \theta)=i_{0}(\theta) \geq 0, \quad a(0, a)=a_{0}(a) \geq 0 .
$$

For the system (2.6) and (2.7), we define the number of the newly infected per unit time $B_{1}(t)$ and the number of new onsets per unit time $B_{2}(t)$ as

$$
B_{1}(t)=i(t, 0), \quad B_{2}(t)=a(t, 0) .
$$

By using the method of characteristic lines, from (2.6) and (2.7) we have the following expression: for $0<t<T, a>0$,

$$
i(t, \theta)= \begin{cases}B_{1}(t-\theta), & t>\theta \\ i_{0}(\theta-t), & t \leq \theta\end{cases}
$$

for $t \in[n T,(n+1) T), n=1,2,3, \ldots, a>0$,

$$
i(t, \theta) \begin{cases}B_{1}(t-\theta), & t-n T>\theta, \\ (1-p) B_{1}(t-\theta), & t-n T \leq \theta<t-(n-1) T, \\ \vdots & \\ (1-p)^{n} B_{1}(t-\theta), & t-T \leq \theta<t, \\ (1-p)^{n} i_{0}(\theta-t), & t \leq \theta,\end{cases}
$$


and for $t \in[0,+\infty)$,

$$
a(t, \tau)= \begin{cases}B_{2}(t-\tau), & t>\tau \\ a_{0}(\tau-t), & t \leq \tau\end{cases}
$$

Substituting (2.10) and (2.11) into the expression of $B_{1}(t)$, and changing the order of the first integration, for $t \in[n T,(n+1) T), n=0,1,2,3, \ldots$, we obtain

$$
\begin{aligned}
B_{1}(t)= & S_{0}(1-p)^{n} \int_{t}^{+\infty} \beta_{1}(\theta) l(\theta) \Gamma_{1}(\theta) \Pi_{1}(\theta) i_{0}(\theta-t) d \theta \\
& +\int_{0}^{t-n T} B_{1}(t-\theta) \beta_{1}(\theta) l(\theta) \Gamma_{1}(\theta) \Pi_{1}(\theta) d \theta \\
& +(1-p) \int_{t-n T}^{t-(n-1) T} B_{1}(t-\theta) \beta_{1}(\theta) l(\theta) \Gamma_{1}(\theta) \Pi_{1}(\theta) d \theta \\
& +\cdots+(1-p)^{n} \int_{t-T}^{t} B_{1}(t-\theta) \beta_{1}(\theta) l(\theta) \Gamma_{1}(\theta) \Pi_{1}(\theta) d \theta \\
& +S_{0} \int_{t}^{+\infty} \beta_{2}(\tau) l(\tau) \Gamma_{2}(\tau) \Pi_{2}(\tau) a_{0}(\tau-t) d \tau+S_{0} \int_{0}^{t} \beta_{2}(\tau) l(\tau) \Gamma_{2}(\tau) \Pi_{2}(\tau) B_{2}(t-\tau) d \tau
\end{aligned}
$$

Inserting (2.10) into the expression of $B_{2}(t)$, we have

$$
\begin{aligned}
B_{2}(t)= & S_{0}(1-p)^{n} \int_{t}^{+\infty} \eta(\theta) l(\theta) \Gamma_{1}(\theta) \Pi_{1}(\theta) i_{0}(\theta-t) d \theta \\
& +\int_{0}^{t-n T} B_{1}(t-\theta) \eta(\theta) l(\theta) \Gamma_{1}(\theta) \Pi_{1}(\theta) d \theta \\
& +(1-p) \int_{t-n T}^{t-(n-1) T} B_{1}(t-\theta) \eta(\theta) l(\theta) \Gamma_{1}(\theta) \Pi_{1}(\theta) d \theta \\
& +\cdots+(1-p)^{n} \int_{t-T}^{t} B_{1}(t-\theta) \eta(\theta) l(\theta) \Gamma_{1}(\theta) \Pi_{1}(\theta) d \theta .
\end{aligned}
$$

Let

$$
h(t, \theta)= \begin{cases}1, & t-n T>\theta \\ (1-p), & t-n T \leq \theta<t-(n-1) T, \\ \vdots & \\ (1-p)^{n}, & t-T \leq \theta<t .\end{cases}
$$


Then $B(t)=\left(B_{1}(t), B_{2}(t)\right)^{T}$ satisfies the renewal integral equations as follows:

$$
\begin{aligned}
B_{1}(t)= & S_{0} \int_{0}^{t} B_{1}(t-\theta) \beta_{1}(\theta) l(\theta) \Gamma_{1}(\theta) \Pi_{1}(\theta) h(t, \theta) d \theta \\
& +S_{0} \int_{0}^{t} B_{2}(t-\tau) \beta_{2}(\tau) l(\tau) \Gamma_{2}(\tau) \Pi_{2}(\tau) d \tau+F_{1}(t), \\
B_{2}(t)= & \int_{0}^{t} B_{1}(t-\theta) \eta(\theta) l(\theta) \Gamma_{1}(\theta) \Pi_{1}(\theta) h(t, \theta) d \theta+F_{2}(t),
\end{aligned}
$$

where

$$
\begin{aligned}
F_{1}(t)= & S_{0}(1-p)^{n} \int_{t}^{+\infty} \beta_{1}(\theta) l(\theta) \Gamma_{1}(\theta) \Pi_{1}(\theta) i_{0}(\theta-t) d \theta \\
& +S_{0} \int_{t}^{+\infty} \beta_{2}(\tau) l(\tau) \Gamma_{2}(\tau) \Pi_{2}(\tau) a_{0}(\tau-t) d \tau \\
F_{2}(t)= & S_{0}(1-p)^{n} \int_{t}^{+\infty} \eta(\theta) l(\theta) \Gamma_{1}(\theta) \Pi_{1}(\theta) i_{0}(\theta-t) d \theta
\end{aligned}
$$

and $\lim _{t \rightarrow+\infty} F_{j}(t)=0(j=1,2)$.

In the following, we discuss the condition of permanence and extinction for HIV / AIDS. For $\tau \in[n T,(n+1) T), n=0,1,2,3, \ldots$, let

$$
H_{1}(\tau)=(1-p) H(\tau), \quad H_{2}(\tau)=H(\tau),
$$

where

$$
H(\tau)= \begin{cases}1, & 0 \leq \tau<T \\ 1-p, & T \leq \tau<2 T \\ \vdots & \\ (1-p)^{n}, & n T \leq \tau<(n+1) T .\end{cases}
$$

From (2.18), we have

$$
H_{1}(\tau) \leq h(t, \tau) \leq H_{2}(\tau), \quad \tau \in[0, t), t \in[0,+\infty) .
$$


Consider the following systems $(j=1,2)$ :

$$
\begin{aligned}
B_{1 j}(t)= & S_{0} \int_{0}^{t} B_{1 j}(t-\theta) \beta_{1}(\theta) l(\theta) \Gamma_{1}(\theta) \Pi_{1}(\theta) H_{j}(\theta) d \theta \\
& +S_{0} \int_{0}^{t} B_{2 j}(t-\tau) \beta_{2}(\tau) l(\tau) \Gamma_{2}(\tau) \Pi_{2}(\tau) d \tau+F_{1}(t), \\
B_{2 j}(t)= & \int_{0}^{t} B_{1 j}(t-\theta) \eta(\theta) l(\theta) \Gamma_{1}(\theta) \Pi_{1}(\theta) H_{j}(\theta) d \theta+F_{2}(t) .
\end{aligned}
$$

Let $\left(B_{1 j}(t), B_{2 j}(t)\right)^{T}(j=1,2)$ be the solutions of integral equations (2.21). From (2.20), we obtain

$$
\left(B_{11}(t), B_{21}(t)\right)^{T} \leq\left(B_{1}(t), B_{2}(t)\right)^{T} \leq\left(B_{12}(t), B_{22}(t)\right)^{T},
$$

where we write $U_{1} \leq U_{2}$, if and only if $U_{2}-U_{1} \in X_{+}, U_{1}, U_{2} \in X_{+}$,

$$
\begin{gathered}
X=L^{1}(0,+\infty) \times L^{1}(0,+\infty) \\
X_{+}=\left\{(u, v)^{T}:(u, v)^{T} \in X, u \geq 0, v \geq 0, \text { a.e. }\right\} .
\end{gathered}
$$

Using the standard argument as in [22], we easily obtain that the threshold condition of systems (2.21) can be formulated by the spectral radius of $K_{j}(j=1,2)$ defined as

$$
K_{j}=\left(\begin{array}{cc}
\alpha_{1 j} & \alpha_{3} \\
\alpha_{2 j} & 0
\end{array}\right)
$$

where

$$
\begin{gathered}
\alpha_{1 j}(p, T)=S_{0} \int_{0}^{+\infty} \beta_{1}(\theta) l(\theta) \Gamma_{1}(\theta) \Pi_{1}(\theta) H_{j}(\theta) d \theta, \\
\alpha_{2 j}(p, T)=\int_{0}^{+\infty} \eta(\theta) l(\theta) \Gamma_{1}(\theta) \Pi_{1}(\theta) H_{j}(\theta) d \theta, \\
\alpha_{3}=S_{0} \int_{0}^{+\infty} \beta_{2}(\tau) l(\tau) \Gamma_{2}(\tau) \Pi_{2}(\tau) d \tau,
\end{gathered}
$$

that is, $\left(B_{1 j}(t), B_{2 j}(t)\right)^{T}(j=1,2)$ is positive if and only if the spectral radius (positive eigenvalue) $r\left(K_{j}\right)=(1 / 2)\left(\alpha_{1 j}+\sqrt{\alpha_{1 j}^{2}+4 \alpha_{2 j} \alpha_{3}}\right)$ of $K_{j}$ is greater than unity.

Therefore, we conclude that:

Proposition 2.1. (1) If $r\left(K_{1}\right)>1$, HIV/AIDS can invade, that is, $\left(B_{1}(t), B_{2}(t)\right)^{T} \geq\left(B_{11}(t), B_{21}(t)\right)^{T}$ $>0$.

(2) If $r\left(K_{2}\right)<1$, HIV/AIDS cannot persist. 


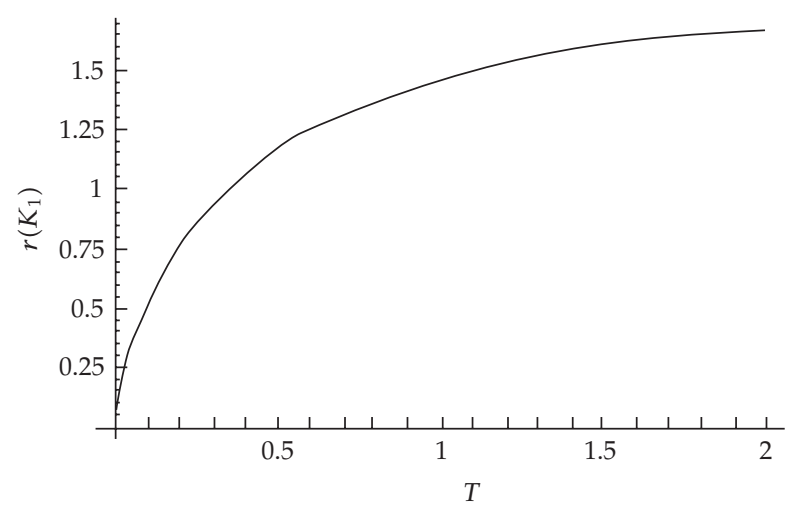

Figure 1: $r\left(K_{1}\right)$ diagram as a function of impulsive period $T$ with parameter values: $S_{0}=1, \mu=0.02$, $\beta_{1}=0.1, \beta_{2}=0.2, \rho=0.5, \eta=0.1, \gamma_{1}=0.2, \gamma_{2}=0.4$, and $p=0.2$.

The conclusion (1) in Proposition 2.1 is epidemiologically important. In fact,

$$
\begin{gathered}
\alpha_{11}(p, T)=S_{0} \sum_{n=0}^{\infty}(1-p)^{n+1} \int_{n T}^{(n+1) T} \beta_{1}(\theta) l(\theta) \Gamma_{1}(\theta) \Pi_{1}(\theta) d \theta, \\
\alpha_{21}(p, T)=\sum_{n=0}^{\infty}(1-p)^{n+1} \int_{n T}^{(n+1) T} \eta(\theta) l(\theta) \Gamma_{1}(\theta) \Pi_{1}(\theta) d \theta .
\end{gathered}
$$

We easily that $r\left(K_{1}\right)=(1 / 2)\left(\alpha_{11}+\sqrt{\alpha_{11}^{2}+4 \alpha_{21} \alpha_{3}}\right)$ increases if the impulsive drug treatment proportion $p$ decreases or impulsive period $T$ increases. So, HIV / AIDS can always invade the host population even if we apply an impulsive drug treatment scheme with the impulsive proportion $p$ and impulsive period $T$, when $p, T$ satisfy $r\left(K_{1}\right)>1$ (see Figure 1 for $p=0.2$ ). The impulsive prevention programme cannot effectively control HIV/AIDS transmission.

\section{Global Stability of the Infection-Free State}

System (2.15) and (2.16) always exists as zero solution, which corresponds to the infectionfree steady state $E_{0}=(0,0)$ of system (2.6) and (2.7).

In the following, we show that the infection-free steady state $E_{0}$ is globally stable, that is, HIV / AIDS goes to extinction. First, we need a lemma, which use the following notion: for any bounded function $f$ on $[0,+\infty)$, let $f^{\infty}=\limsup _{t \rightarrow+\infty} f(t)$.

Lemma 3.1 (see [18]). If $f(t)$ is bounded on $[0,+\infty), g(t) \in L^{1}(0,+\infty)$, then

$$
\limsup _{t \rightarrow+\infty} \int_{0}^{t} f(\theta) g(t-\theta) d \theta \leq\|g\|_{L^{1}(0,+\infty)} f^{\infty}
$$


Noting that $h(t, \theta) \leq H_{1}(\theta)$, and inserting (2.16) into (2.15), we have

$$
\begin{aligned}
B_{1}(t) \leq & S_{0} \int_{0}^{t} B_{1}(t-\theta) \beta_{1}(\theta) l(\theta) \Gamma_{1}(\theta) \Pi_{1}(\theta) H_{1}(\theta) d \theta \\
& +S_{0} \int_{0}^{t} \beta_{2}(\tau) l(\tau) \Gamma_{2}(\tau) \Pi_{2}(\tau) \int_{0}^{t-\tau} B_{1}(t-\tau-\theta) \eta(\theta) l(\theta) \Gamma_{1}(\theta) \Pi_{1}(\theta) H_{1}(\theta) d \theta d \tau \\
& +F_{3}(t) \\
= & \int_{0}^{t} P(\theta) B_{1}(t-\theta) d \theta+F_{3}(t)
\end{aligned}
$$

where

$$
\begin{aligned}
F_{3}(t)= & F_{1}(t)+S_{0} \int_{0}^{t} \beta_{2}(\tau) l(\tau) F_{2}(t-\tau) d \tau, \quad \lim _{t \rightarrow+\infty} F_{3}(t)=0, \\
P(\theta)= & S_{0} \beta_{1}(\theta) l(\theta) \Gamma_{1}(\theta) \Pi_{1}(\theta) H_{1}(\theta) \\
& +S_{0} \int_{0}^{\theta} \beta_{2}(\tau) l(\tau) \Gamma_{2}(\tau) \Pi_{2}(\tau) \eta(\theta-\tau) l(\theta-\tau) \Gamma_{1}(\theta-\tau) \Pi_{1}(\theta-\tau) H_{1}(\theta-\tau) d \tau .
\end{aligned}
$$

Using Lemma 3.1, from (3.2) we have

$$
\begin{aligned}
B_{1}^{\infty} \leq & S_{0} \int_{0}^{+\infty} \beta_{1}(\theta) l(\theta) \Gamma_{1}(\theta) \Pi_{1}(\theta) H_{1}(\theta) d \theta \\
& +\int_{0}^{+\infty} \beta_{2}(\tau) l(\tau) \Gamma_{2}(\tau) \Pi_{2}(\tau) d \tau \int_{0}^{+\infty} \eta(\theta) l(\theta) \Gamma_{1}(\theta) \Pi_{1}(\theta) H_{1}(\theta) d \theta B_{1}^{\infty} \\
= & \left(\alpha_{11}+\alpha_{21} \alpha_{3}\right) B_{1}^{\infty} .
\end{aligned}
$$

Let $R_{1}(p, T)=\left(\alpha_{11}+\alpha_{21} \alpha_{3}\right)\left(\alpha_{11}, \alpha_{21}, \alpha_{3}\right.$ were defined in Section 2$)$. From (3.4), it is easy to see that $B_{1}^{\infty}=0$ if $R_{1}(p, T)<1$.

From (2.10), $B_{1}^{\infty}=0$ implies that

$$
\lim _{t \rightarrow+\infty} i(t, \theta)=0
$$

Using Lemma 3.1 and (2.16), we obtain

$$
B_{2}^{\infty} \leq \int_{0}^{+\infty} \eta(\theta) l(\theta) \Gamma_{1}(\theta) \Pi(\theta) H_{1}(\theta) d \theta B_{1}^{\infty}=\alpha_{21}(p, T) B_{1}^{\infty} .
$$

If $R_{1}(p, T)<1$ and $\alpha_{21}(p, T)<1$, then $B_{2}^{\infty}=0$, which implies that, from (2.11),

$$
\lim _{t \rightarrow+\infty} a(t, \tau)=0 .
$$

From (3.5) and (3.7), we conclude what follows. 


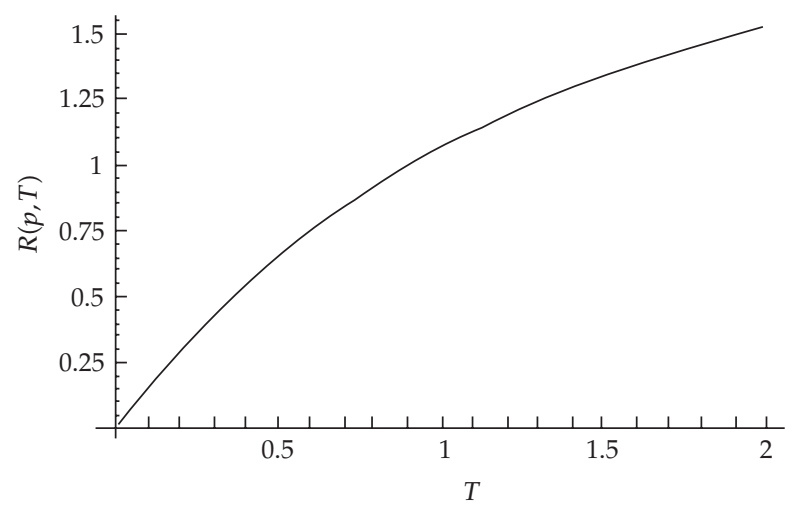

Figure 2: $R(p, T)$ diagram as a function of impulsive period $T$ with parameter values: $S_{0}=1, \mu=0.02$, $\beta_{1}=0.1, \beta_{2}=0.2, \rho=0.5, \eta=0.1, \gamma_{1}=0.2, \gamma_{2}=0.4, p=0.6$.

Theorem 3.2. If $R_{1}(p, T)<1$ and $\alpha_{21}(p, T)<1$ hold, then the infection-free steady state $E_{0}$ is globally stable, that is,

$$
\lim _{t \rightarrow+\infty}(i(t, \theta), a(t, \tau))=(0,0)
$$

Let $R(p, T)=\max \left\{R_{1}(p, T), \alpha_{21}(p, T)\right\}$. From the expression of $R_{1}(p, T)$ and $\alpha_{21}(p, T)$, it is easy to see that $R_{1}(p, T), \alpha_{3}(p, T)$ decreases if the impulsive drug treatment proportion $p$ increases or impulsive period $T$ decreases. Hence, we can choose $p(0<p<1)$ and $T$ such that $R(p, T)<1$ (see Figure 2), which implies that HIV/AIDS will be vastly reduced in real life.

\section{Discussion}

In this paper, our disease-control strategy is targeted at infected individuals ( $I$ control); we investigate the effects of impulsive period $T$ and impulsive drug treatment proportion $p$ for HIV / AIDS transmission. The results would provide an idea to estimate optimal pulse interval and impulsive proportion.

The dynamics of the spread of HIV/AIDS is so complex that we could study it all at once. In this paper, for simplicity we do not consider that HIV / AIDS transmission depends on individuals' chronological age. In fact, the most HIV / AIDS cases $(23 / 100)$ are in the age group of 40-44 years. AIDS transmission rate $\beta_{2}$ is given as a function of the disease-age (i.e., the time elapsed since the onset). However, because the infectiousness might be in part characterized by the density of pathogens in vivo, there is a possibility that $\beta_{2}$ depends on the infection age (i.e., the time since infection).

Biologically speaking, if the size of infected individuals is very large, then treating the same proportion of infected people every few months is unrealistic. In addition, the proportion $p$ would change in real life, but for theoretical analysis, we assume that it is a constant. 


\section{References}

[1] O. Krakovska and L. M. Wahl, "Optimal drug treatment regimens for HIV depend on adherence," Journal of Theoretical Biology, vol. 246, no. 3, pp. 499-509, 2007.

[2] http://www.who.int/hiv/topics/treatment/en/.

[3] S. Busenberg and C. Castillo-Chavez, "A general solution of the problem of mixing of subpopulations and its application to risk- and age-structured epidemic models for the spread of AIDS," Mathematical Medicine and Biology, vol. 8, no. 1, pp. 1-29, 1991.

[4] J. M. Hyman, J. Li, and E. A. Stanley, "Threshold conditions for the spread of the HIV infection in agestructured populations of homosexual man," Journal of Theoretical Biology, vol. 166, no. 1, pp. 9-31, 1994.

[5] H. Inaba, "Endemic threshold results in an age-duration-structured population model for HIV infection," Mathematical Biosciences, vol. 201, no. 1-2, pp. 15-47, 2006.

[6] R. M. May, R. M. Anderson, and A. R. McLean, "Possible demographic consequences of HIV / AIDS," Mathematical Biosciences, vol. 90, no. 1, pp. 475-506, 1988.

[7] R. M. May and R. M. Anderson, "Transmission dynamics of HIV infection," Nature, vol. 326, no. 6019, pp. 137-142, 1987.

[8] J. A. P. Heesterbeek and M. G. Roberts, "The type-reproduction number $T$ in models for infectious disease control," Mathematical Biosciences, vol. 206, no. 1, pp. 3-10, 2007.

[9] V. Lakshmikantham, D. D. Bainov, and P. S. Simeonov, Theory of Impulsive Differential Equations, World Scientific, Singapore, 1989.

[10] Z. Agur, L. Cojocaru, G. Mazor, R. M. Anderson, and Y. L. Danon, "Pulse mass measles vaccination across age cohorts," Proceedings of the National Academy of Sciences of the United States of America, vol. 90, no. 24, pp. 11698-11702, 1993.

[11] A. D'Onofrio, "Stability properties of pulse vaccination strategy in SEIR epidemic model," Mathematical Biosciences, vol. 179, no. 1, pp. 57-72, 2002.

[12] Y. Zhou and H. Liu, "Stability of periodic solutions for an SIS model with pulse vaccination," Mathematical and Computer Modelling, vol. 38, no. 3-4, pp. 299-308, 2003.

[13] M. Ramsay, N. Gay, E. Miller, et al., "The epidemiology of measles in England and Wales: rationale for the 1994 national vaccination campaign," Communicable Disease Report, vol. 4, no. 12, pp. 141-146, 1994.

[14] R. J. Smith and 1. M. Wahl, "Distinct effects of protease and reverse transcriptase inhibition in an immunological model of HIV-1 infection with impulsive drug effects," Bulletin of Mathematical Biology, vol. 66, no. 5, pp. 1259-1283, 2004.

[15] R. J. Smith and L. M. Wahl, "Drug resistance in an immunological model of HIV-1 infection with impulsive drug effects," Bulletin of Mathematical Biology, vol. 67, no. 4, pp. 783-813, 2005.

[16] H. R. Thieme, "Semiflows generated by lipschitz perturbations of non-densely defined operators," Differential and Integral Equations, vol. 3, no. 6, pp. 1035-1066, 1990.

[17] M. Martcheva and H. R. Thieme, "Progression age enhanced backward bifurcation in an epidemic model with super-infection," Journal of Mathematical Biology, vol. 46, no. 5, pp. 385-424, 2003.

[18] Z. Feng, M. Iannelli, and F. A. Milner, "A two-strain tuberculosis model with age of infection," SIAM Journal on Applied Mathematics, vol. 62, no. 5, pp. 1634-1656, 2002. 


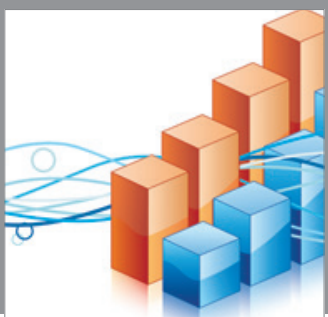

Advances in

Operations Research

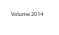

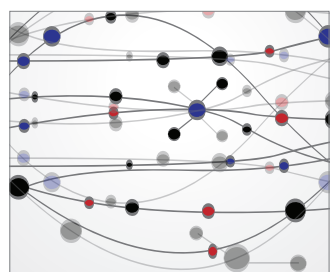

\section{The Scientific} World Journal
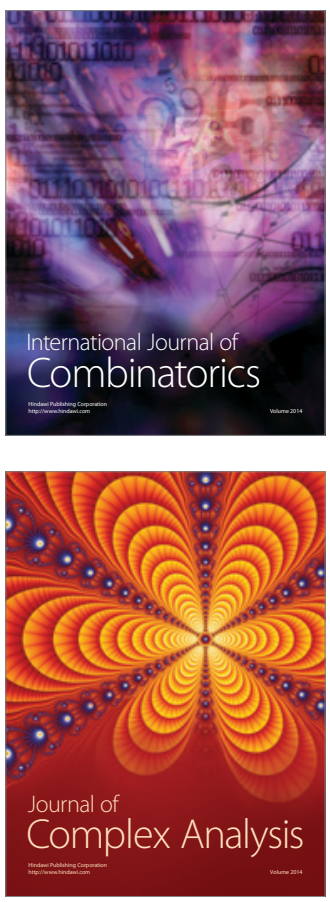

International Journal of

Mathematics and

Mathematical

Sciences
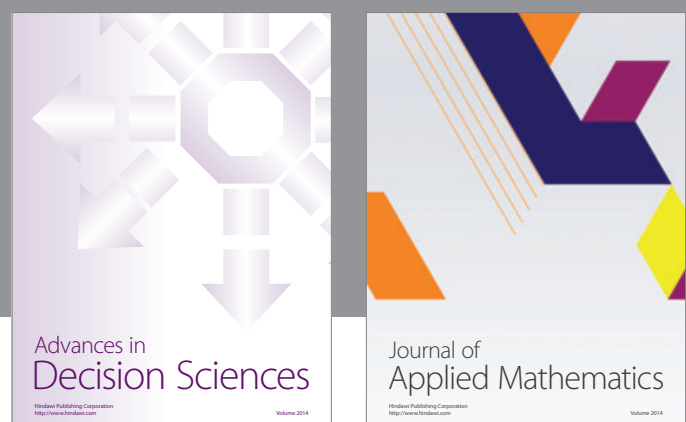

Journal of

Applied Mathematics
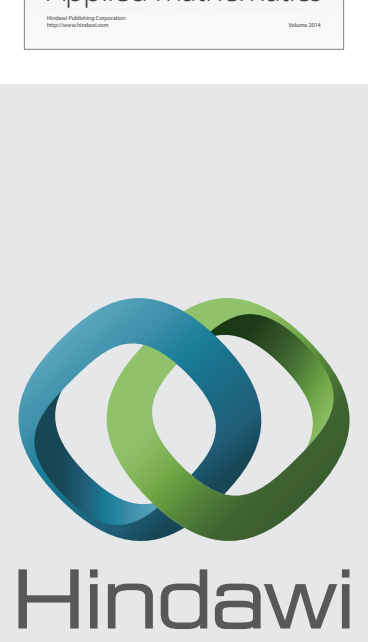

Submit your manuscripts at http://www.hindawi.com
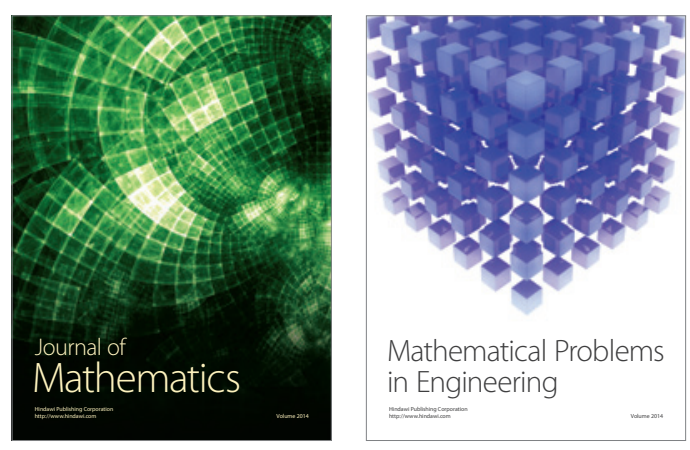

Mathematical Problems in Engineering
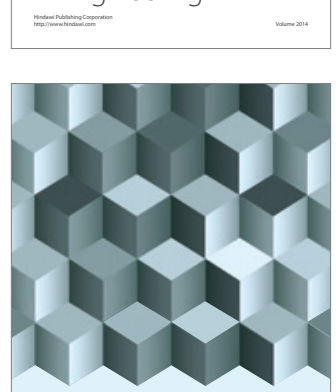

Journal of

Function Spaces
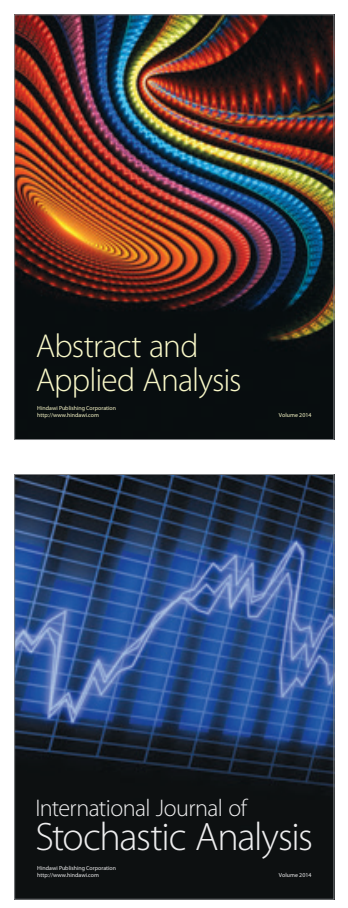

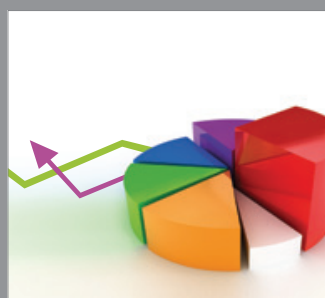

ournal of

Probability and Statistics

Promensencen
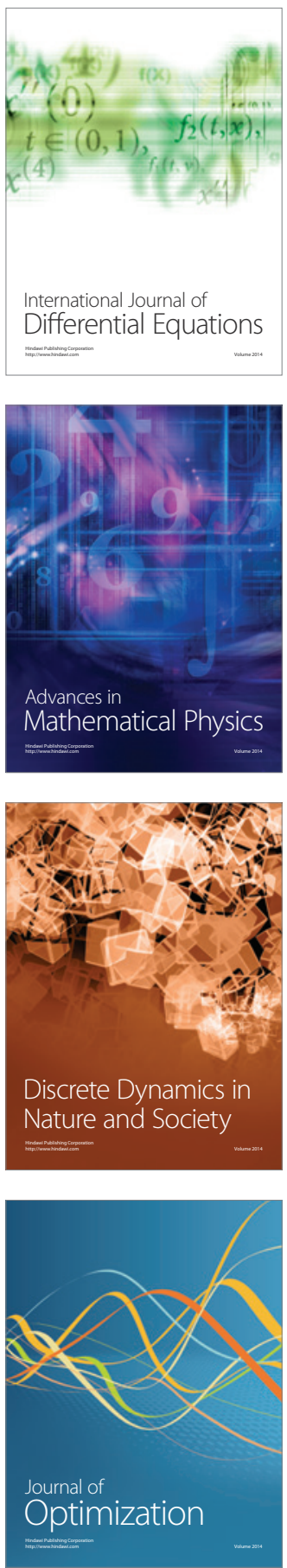